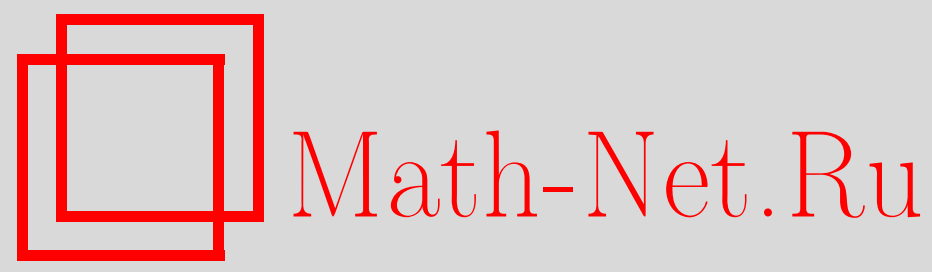

Р. И. Подловченко, В. Е. Хачатрян, Полное решение проблемы минимизации для одного множества бинарных двухленточных автоматов, Дискрет. матем., 2010, том 22, выпуск $3,146-159$

DOI: https://doi.org/10.4213/dm1114

Использование Общероссийского математического портала Math-Net.Ru подразумевает, что вы прочитали и согласны с пользовательским соглашением http://www . mathnet.ru/rus/agreement

Параметры загрузки:

IP : 54.210 .77 .194

26 апреля 2023 г., $17: 37: 12$ 


\title{
Полное решение проблемы минимизации для одного множества бинарных двухленточных автоматов
}

\author{
( 2010 г. Р. И. Подловченко, В. Е. Хачатрян
}

В статье завершается решение проблемы минимизации для одного множества бинарных двухленточных автоматов. Начало решения было опубликовано в журнале «Дискретная математика» в 2008 г.

Проблема минимизации состоит в нахождении всех минимальных автоматов в каждом классе эквивалентности из рассматриваемого множества. Поиск осуществляется эквивалентными преобразованиями автоматов. Методика решения проблемы основана на подходящем разбиении класса эквивалентности.

Работа выполнена при поддержке Российского фонда фундаментальных исследований, проект 06-01-00106.

\section{1. Введение}

Данная статья является продолжением статьи [1], в которой изложена первая часть решения следующей задачи: для конкретного множества детерминированных бинарных двухленточных автоматов, обозначаемого $M$, построить процедуру отыскания всех минимальных по размеру автоматов в любом его классе эквивалентности. Эта задача названа в [1] обобщенной проблемой минимизации в $M$ в знак того, что предварительно в $M$ были обнаружены классы эквивалентности с несколькими минимальными в них автоматами.

Для краткости мы будем называть эту задачу просто проблемой минимизации.

В [1] проблема минимизации в $M$ сведена к проблеме минимизации в $\bar{M}$, где $\bar{M}-$ некоторое подмножество множества $M$. Именно для $\bar{M}$ и получены в [1] многие результаты, относящиеся к решению в нем проблемы минимизации.

В данной статье рассматривается тоже множество $\bar{M}$.

Для решения выдвинутой проблемы в [1] любой класс эквивалентности $K$ в $\bar{M}$ разбивается на попарно непересекающиеся подмножества, называемые срезами класса $K$. Среди них выделен срез, называемый главным, и статья [1] посвящена отысканию всех минимальных автоматов в главном срезе.

Здесь мы покажем, что в общем случае минимальные автоматы в главном срезе не являются минимальными в классе $K$. Это и приводит к необходимости рассматривать срезы, отличные от главного. Известный размер минимальных автоматов в главном срезе сразу устанавливает, что число срезов, подлежащих исследованию, конечно.

В данной статье, в первую очередь, доказывается, что минимальным в $K$ может быть только тупиковый автомат, то есть не имеющий отличающихся друг от друга эквивалентных состояний (см. лемму 1). 
В главном срезе класса $K$ имеется минимальный автомат, обладающий тем свойством, что все другие минимальные в этом срезе автоматы конструируются по нему эффективно; этот автомат назван в [1] основным, обозначим его $A_{0}$.

Очевидным образом выделяются преобразования, применяемые к автомату $A_{0}$ и транслирующие его в автоматы из других срезов класса $K$, какими бы эти срезы ни были (см. предложение 1). Эти преобразования разложимы в цепочки в определенном смысле атомарных преобразований. В связи с этим возникает задача выявить атомарные преобразования, посредством которых можно попасть в срезы с тупиковыми автоматами.

Решение этой задачи проводится нами в случае, когда атомарные преобразования применяются к тупиковому автомату, не обязательно совпадающему с $A_{0}$, но обладающему важным свойством, присущим автомату $A_{0}$, такой автомат назван перспективным.

Основные результаты, полученные нами, излагаются в следующем порядке. Сначала выделяются те и только те атомарные преобразования, которыми перспективный автомат транслируется в автомат, преобразуемый в тупиковый, не покидая полученного среза (см. лемму 2); считаем его базовым в своем срезе, а выделенные атомарные преобразования именуем правильными. Затем строится процедура трансформации базового автомата в минимальный в том же срезе, которому принадлежит базовый автомат (см. лемму 3). Оказывается, что построенный минимальный автомат является не только перспективным, но и обладающим следующим свойством: все другие минимальные автоматы в данном срезе строятся по нему эффективно. Он назван стержневым в своем срезе.

Полученными результатами подготовлен главный результат: предложена процедура отыскания всех минимальных автоматов в классе $K$ (см. теорему 1$)$. Эта процедура состоит из двух этапов. На первом рассматриваются стержневые автоматы, и на каждом шаге процедуры фиксируется список наименьших по размеру стержневых автоматов, полученных к началу этого шага. Этап завершается созданием окончательного списка. На втором этапе для всякого автомата, попавшего в этот список, конструируются другие минимальные автоматы.

Итак, в множестве $\bar{M}$ проблема минимизации решена. Ее решение в множестве $M$ обеспечено процедурой сведения проблемы из $M$ в $\bar{M}$ (см. следствие 2).

Статья, кроме введения, имеет четыре раздела. Второй раздел посвящен описанию множеств $M$ и $\bar{M}$, сжатому изложению применяемого нами аппарата фрагментных преобразований автоматов (см. [2]) и краткому обзору основных фактов, установленных в [1]. Этим предусматривается минимум сведений, обеспечивающих сравнительно независимое от [1] чтение данной статьи. Третий раздел начинается примером случая, когда основной автомат не является минимальным в своем классе эквивалентности, и содержит доказательство леммы 1. В четвертом разделе изучаются атомарные преобразования и доказывается лемма 2. В пятом разделе доказана лемма 3 и установлены факты, обеспечивающие корректность предлагаемой нами процедуры отыскания всех минимальных автоматов в классе $K$ из $\bar{M}$. Сама процедура изложена в теореме 1 .

Отметим, что в статье [3] рассмотрен случай, когда основной автомат $A_{0}$ является минимальным в классе $K$.

\section{2. Изучаемые множества бинарных автоматов и результаты, полученные ранее}

Сначала опишем упомянутое во введении множество $M$ автоматов. Оно состоит из детерминированных бинарных двухленточных автоматов, структура каждого из которых 
описывается следующим образом. Это конечный ориентированный граф, вершины которого называются состояниями. Среди них обязательно присутствует три выделенные состояния: инициальное, финальное и мертвое. Инициальное состояние не имеет входящих в него дуг, финальное и мертвое не имеют исходящих из них дуг. Любое состояние, кроме мертвого, помечается либо символом $p$ и называется $p$-состоянием, либо символом $q$ и называется $q$-состоянием. Из каждого состояния, кроме финального и мертвого, исходят две дуги, помеченные числами 1 и 0 соответственно. Из любого $q$-состояния дуга с меткой 1 ведет в мертвое состояние. Всякое состояние автомата, кроме мертвого, находится на ориентированном пути из инициального состояния в финальное, этот путь называется путем через автомат.

Произвольному пути через автомат сопоставляется упорядоченная пара цепочек из нулей и единиц; первая цепочка строится выписыванием меток дуг, исходящих из p-состояний, при просмотре их в пути от его начала к концу, вторая цепочка получается аналогичным способом при просмотре $q$-состояний, принадлежащих пути.

Два автомата из $M$, по определению, эквивалентны, если для любого пути через один из них существует путь через другой автомат, характеризующийся той же парой цепочек, что и первый.

Как было отмечено во введении, проблема минимизации в $M$ сводится к проблеме минимизации в $\bar{M}$; здесь $\bar{M}$ состоит из автоматов, в которых имеются как $p$-состояния, так и $q$-состояния. Этот результат получен предварительным построением системы $T$ эквивалентных преобразований (э.п.), полной в $M$ (см. теорему 1 из [1]). Под полнотой системы понимается следующее ее свойство: какими бы ни были два эквивалентных автомата из $M$, существует конечная цепочка э.п. из $T$, транслирующая первый автомат во второй.

Система $T$ компонуется из эквивалентных фрагментных преобразований автоматов. Опишем кратко, как строится система $T$.

Фрагментом автомата называется его часть, порожденная некоторым множеством его состояний и всеми инцидентными им дугами; состояния и дуги наследуют метки, присвоенные им в автомате. Связь фрагмента с остальной частью автомата задается множеством приходящих в него дуг из состояний, не принадлежащих фрагменту, и множеством исходящих из него дуг, ведущих в состояния, ему не принадлежащие. Начало и конец каждой приходящей дуги называются внешним и внутренним входами фрагмента, а исходящей - внутренним и внешним выходами фрагмента.

В [1] описана операция подстановки вместо какого-либо фрагмента автомата другого фрагмента. Она транслирует данный автомат из $M$ в автомат из $M$ и называется фрагментным преобразованием. Любая пара фрагментов, допускающих операцию подстановки вместо одного из них другого, определяет множество фрагментных преобразований. Если все они являются эквивалентными, то фрагменты называются эквивалентными.

Система $T$ индуцируется двумя разрешимыми множествами, состоящими из пар эквивалентных фрагментов; эти множества именуются аксиомами $B 1$ и $B 2$. Замена первого в паре фрагмента вторым называется преобразованием $B i \uparrow$, обратная замена - преобразованием $B i \downarrow, i=1,2$.

Доказательство полноты системы $T$ проводится традиционным образом, а именно, построением в каждом классе эквивалентности $K$ в $M$ единственного (с точностью до изоморфизма) его представителя, названного каноническим в $K$ автоматом.

Все проводимые преобразования автоматов из $M$ и $\bar{M}$ - это преобразования из системы $T$. Как мы увидим далее, при поиске минимальных автоматов канонический автомат не используется. Это позволяет опустить его определение. Решение проблемы минимизации в $\bar{M}$ базируется на разбиении класса $K$ на попарно непересекающиеся подмножества, 
называемые его срезами. Отдельный срез состоит из всех автоматов класса $K$, имеющих общую $p$-проекцию. Последняя строится однозначно по автомату корректным устранением в нем всех $q$-состояний и представляет собой, в сущности, обычный конечный автомат.

В [1] описана подсистема $T_{0}$ системы $T$, являющаяся полной в любом срезе класса $K$ (см. предложение 10).

Значительная часть статьи [1] посвящена исследованию главного среза класса $K$. Так называется срез, $p$-проекция которого минимальна как обычный конечный автомат. В [1] предложена процедура построения минимальных автоматов в главном срезе класса $K$. Сначала конструируется минимальный автомат, названный основным, затем описываются преобразования, посредством которых можно получить из основного все другие минимальные автоматы в главном срезе.

Предложенная процедура активно использует так называемое $\varphi$-преобразование. Остановимся на содержательном его описании. Оно представляет собой цепочку преобразований из системы $T_{0}$, применяемую к фрагменту автомата, называемому $s$-фрагментом. Последним является фрагмент, в котором внутренние его входы - это либо $q$-состояния, либо ведущие непосредственно в $q$-состояния; первые называются активными входами, а вторые пассивными. Внешний выход $s$-фрагмента называется активным, если в него не приходят извне фрагмента дуги из $q$-состояний, и пассивным в ином случае. Посредством $\varphi$-преобразования $s$-фрагмент трансформируется в фрагмент, в котором все его внутренние выходы - это q-состояния; он называется фрагментом с нижней границей.

Если в $s$-фрагменте число его активных входов превышает число его активных выходов, то $\varphi$-преобразование $s$-фрагмента уменьшает число принадлежащих автомату $q$-состояний и называется успешным; при равенстве числа активных входов и активных выходов число $q$-состояний в преобразуемом автомате сохраняется неизменным, и тогда $\varphi$-преобразование называется устойчивым.

Отметим, что конструирование основного автомата совершается только успешными $\varphi$-преобразованиями, а получение других минимальных автоматов в главном срезе устойчивыми $\varphi$-преобразованиями основного автомата.

\section{3. Предварительно устанавливаемые факты}

Начнем с примера, демонстрирующего случай, когда основной автомат не является минимальным в своем классе эквивалентности $K$.

На рис. 1 представлены автоматы $A_{0}, A_{1}, A_{2}, A_{3}, A_{4}$; все они принадлежат множеству $\bar{M}$. При изображении структуры автомата нами используются следующие соглашения. Состояния автомата маркируются символами $q$ и $p$, будучи лишены своих обозначений. Дуга, помеченная символом 1 и исходящая из $p$-состояния, снабжается жирной точкой у ее основания, что позволяет не выставлять метки дуг. Мертвое состояние автомата вместе с приходящими в него дугами не изображается; инициальное состояние выделено затемненным кружочком, финальное - кружочком с крестиком.

На рис. 1 в каждом из представленных автоматов заштрихованным прямоугольником выделен фрагмент, рассматриваемый при трансформации этого автомата в автомат со следующим номером. Легко увидеть, что все выделенные фрагменты индуцируются изоморфными множествами состояний, если не учитывать приходящих в них и исходящих из них дуг. Тем не менее, за выделенным фрагментом закрепим номер, присвоенный самому автомату. 


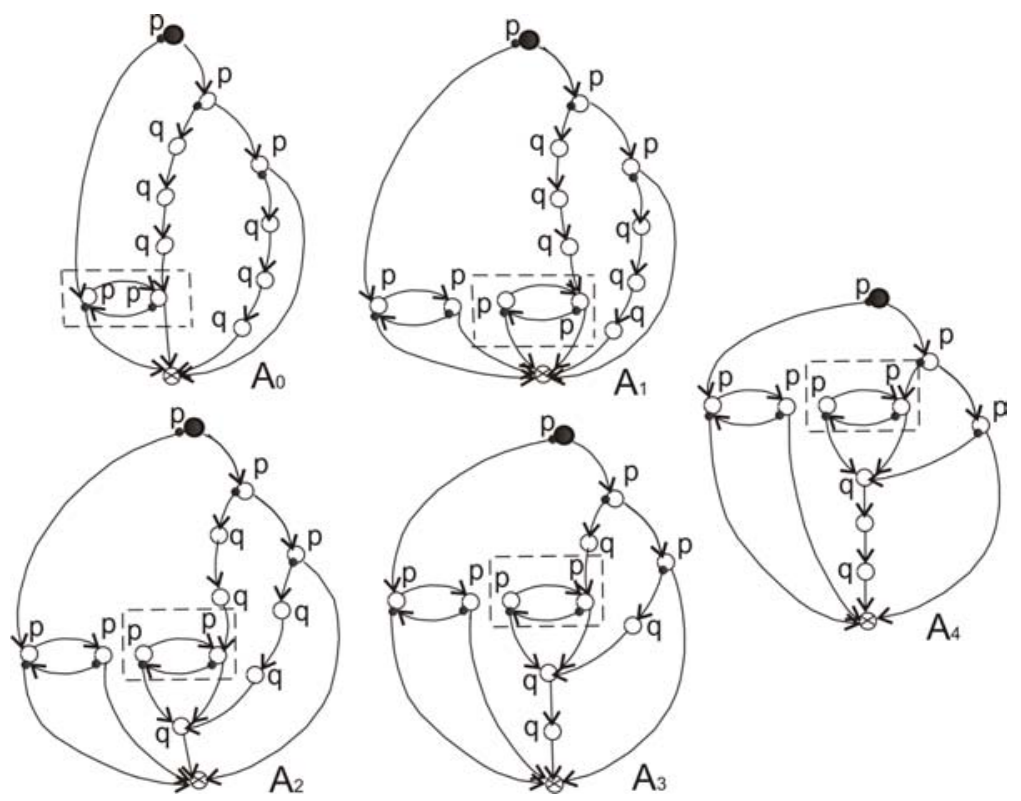

Pис. 1.

Автомат $A_{0}$, как легко убедиться, имеет минимальную $p$-проекцию. Следовательно, $A_{0}$ - автомат из главного среза класса $K$. Поскольку $A_{0}$ не содержит $s$-фрагментов вообще, он является основным автоматом.

Автомат $A_{1}$ получен применением преобразования $B 1 \uparrow$ (оно описано в [1]) к фрагменту $F_{0}$ автомата $A_{0}$. Этим преобразованием $F_{0}$ расклеен на два фрагмента, один из которых и есть $F_{1}$. Автомат $A_{1}$ принадлежит срезу класса $K$, отличному от главного, и не является тупиковым. Фрагмент $F_{1}$ является подфрагментом $s$-фрагмента, обозначим его $\bar{F}_{1}$.

У фрагмента $\bar{F}_{1}$ имеется всего один внутренний вход, причем активный, и всего один внутренний выход, который является пассивным. Таким образом, к фрагменту $\bar{F}_{1}$ применимо успешное $\varphi$-преобразование, оно трансформирует автомат $A_{1}$ в автомат $A_{2}$.

В автомате $A_{2}$ его фрагмент $F_{2}$ является опять-таки подфрагментом $s$-фрагмента, к которому применимо успешное $\varphi$-преобразование, трансформирующее $A_{2}$ в $A_{3}$.

Описанная ситуация повторяется и дальше, в результате чего успешным $\varphi$-преобразованием автомат $A_{3}$ перейдет в автомат $A_{4}$.

Нетрудно проверить, что автоматы $A_{2}, A_{3}$ и $A_{4}$ являются тупиковыми и принадлежат тому же срезу, что и автомат $A_{1}$. В этом срезе $A_{4}$ является минимальным, ибо к нему неприменимы успешные $\varphi$-преобразования. Вместе с тем, автомат $A_{4}$ по размеру меньше автомата $A_{0}$, то есть $A_{0}$ не является минимальным в классе $K$.

Пусть $K-$ рассматриваемый далее класс эквивалентности в множестве $\bar{M}$. Справедливо следующее утверждение.

Лемма 1. Минимальный автомат в классе $K$ является тупиковым.

Доказательство. Предположим, что автомат $A$ - минимальный автомат в $K$, не являющийся тупиковым. Покажем, что в $K$ существует автомат, который размером меньше автомата $A$. 


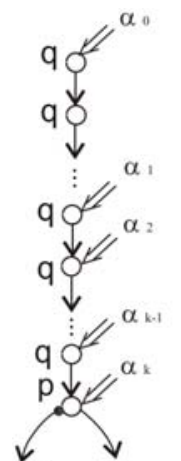

(1) (2)

$F_{1}$

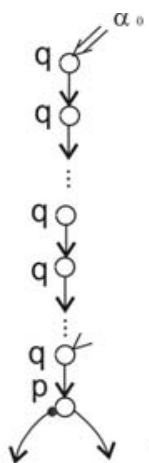

(1)

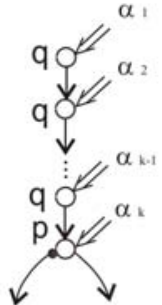

(2)

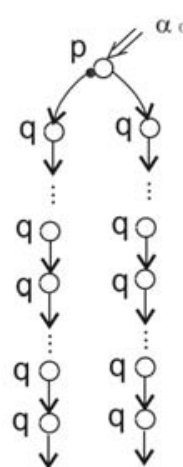

(1)
(2)

$\mathrm{F}_{2} \quad \mathrm{~F}_{3}$

Рис. 2.

Пусть $a$ и $b-$ эквивалентные состояния в $A$. Если $a$ и $b$ однотипны, то выполним операцию склеивания состояния $b$ с состоянием $a$. Она состоит в перебрасывании на состояние $a$ всех приходящих в $b$ дуг, после чего устраняются состояние $b$ и все те состояния, отличные от финального и мертвого, которые стали недостижимы из инициального состояния ориентированными путями. Легко видеть, что выполненная операция трансформирует $A$ в эквивалентный ему автомат, и последний имеет меньший размер, чем автомат $A$.

Рассмотрим теперь случай, когда состояния $a$ и $b$ разнотипны. Для определенности полагаем, что $a$ - это $p$-состояние, а $b$ - это $q$-состояние. Найдем ближайшее $p$-состояние, достижимое из $b$ ориентированным путем $w$. В общем случае минимальный фрагмент, содержащий путь $w$, изображен на рис. 2 как фрагмент $F_{1}$. Здесь двойными стрелками зафиксированы дуги, приходящие в состояние $b$ и остальные состояния пути $w$; при этом отсутствие двойной стрелки означает, что наверняка множество приходящих дуг пусто; метка $\alpha_{1}$ присвоена непустому множеству дуг, а метки $\alpha_{2}, \ldots, \alpha_{k}$ присвоены возможно и пустым множествам дуг; внешние выходы фрагмента $F_{1}$ обозначены (1) и (2); непустое множество дуг, входящих в состояние $b$, помечено $\alpha_{0}$.

На рис. 2 представлен результат расклейки фрагмента $F_{1}$ на фрагменты $F_{2}$ и $F_{3}$, а фрагмент $F_{4}$ получен из фрагмента $F_{2}$ очевидным эквивалентным преобразованием. В фрагменте $F_{4}$ внутренним входом является $p$-состояние, обозначим его $b^{\prime}$. Очевидно, что $b^{\prime}$ эквивалентно состоянию $a$, то есть может быть склеено с ним по правилам, описанным выше. Полученный при этом автомат эквивалентен исходному автомату $A$, но меньше его по размеру.

Отметим, что все частные ситуации легко извлекаются из описанного выше случая разнотипности состояний $a$ и $b$.

Лемма 1 доказана.

Итак, нам предстоит найти срезы класса $K$, отличные от главного и содержащие тупиковые автоматы. Прежде всего, приведем следующее утверждение.

Предложение 1. Каким бы ни был срез класса $K$, преобразованием B1个, примененным к основному в К автомату, можно получить автомат, принадлежащий этому срезу. 
Действительно, как было сказано в разделе 1, -проекция основного автомата - это обычный тупиковый конечный автомат. Это означает, что любая $p$-проекция может быть получена из нее преобразованием $B 1 \uparrow$, ибо она представляет собой просто конечный автомат. Отсюда выводим, что нужно исследовать $B 1 \uparrow$-преобразования.

Всякое такое преобразование разложимо в цепочку атомарных преобразований. К ним относятся преобразования трех типов: раскручивание некоторого цикла в автомате; увеличение его кратности; копирование фрагмента автомата.

Первое преобразование состоит в снятии нескольких витков цикла и, может быть, части витка цикла; второе - в построении нового цикла, виток которого кратен витку исходного. Третье преобразование рассмотрим позже.

Не покидая исследуемого среза, можно выполнять только преобразования из системы $T_{0}$, а это отвергает целесообразность выполнения первого и второго из атомарных преобразований. Действительно, получаемый посредством их нетупиковый автомат невозможно трансформировать в тупиковый средствами системы $T_{0}$, а претенденты на роль минимальных в $K$ автоматов, как это следует из леммы 1 , обязаны быть тупиковыми.

Займемся преобразованием копирования фрагмента $F$ автомата. Оно состоит в построении по фрагменту $F$ фрагментов $F_{1}$ и $F_{2}$, где $F_{1}$ имеет в точности те же состояния, что и $F$, а $F_{2}$ состоит из копий состояний, принадлежащих фрагменту $F$, следовательно, и фрагменту $F_{1}$. При копировании фрагмента $F$ его приходящие дуги распределяются на приходящие в $F_{1}$ и приходящие в $F_{2}$. Это распределение, согласно $B 1 \uparrow$-преобразованию, должно удовлетворять требованию: всякое состояние как в $F_{1}$, так и в $F_{2}$ должно находиться на пути через этот фрагмент. Только тогда копирование фрагмента $F$ выполнено корректно.

Операцию копирования фрагмента $F$ автомата $A$ будем называть операцией $\tau$, полученный ею автомат обозначать $\tau(A, F)$, а срез, которому он принадлежит, обозначать $\sigma(A, F)$. Исследованию операции $\tau$ посвящается раздел 4.

\section{4. Исследование операции копирования фрагмента}

Пусть $A-$ автомат из $\bar{M}$ и $F-$ его фрагмент. Проанализируем операцию $\tau$, примененную к $A$ и $F$.

Прежде всего, отметим следующее. Фрагмент $F$ должен содержать $p$-состояние, иначе срез $\sigma(A, F)$ не отличается от среза, которому принадлежит автомат $A$. Далее, внутренние выходы фрагмента $F$ должны быть $p$-состояниями, поскольку присоединение к $F$ в качестве внутреннего выхода $q$-состояния не приводит к срезу, отличному от $\sigma(A, F)$.

Без дополнительных упоминаний будем предполагать выполненными эти естественные требования к фрагменту $F$.

Говорим, что фрагменту $F$ принадлежит особая цепочка, если в нем существует подфрагмент, состояния которого образуют цепочку, удовлетворяющую следующим требованиям: цепочка начинается $q$-состоянием, завершается $p$-состоянием, которое служит внутренним выходом фрагмента $F$, и если содержит другие состояния, то только $q$-состояния и ни одно состояние, кроме начального, не является внутренним входом подфрагмента.

Операцию $\tau$, примененную к $A$ и $F$, назовем правильной, если фрагмент $F_{2}$ содержит в точности по одной копии состояний из $F_{1}$, а присоединение к фрагменту $F_{2}$ его внешних входов образует $s$-фрагмент, не допускающий своего увеличения за счет внешних входов фрагмента $F_{1}$.

Заметим, что в разделе 5 устанавливается распознаваемость того, допускает ли фрагмент $F$ правильную операцию $\tau$ (см. лемму 4 ). 
Тупиковый автомат $A$ назовем перспективным, если в нем отсутствуют фрагменты с нижней границей, которые не являются результатом успешных $\varphi$-преобразований, и отсутствуют $s$-фрагменты, допускающие успешные $\varphi$-преобразования. Обладающий этими свойствами основной в $K$ автомат подтверждает факт существования перспективных автоматов.

Докажем следующее утверждение.

Лемма 2. Срез $\tau(A, F)$, где $A-$ перспективный автомат, а $F$ - его фрагмент, содержит тупиковые автоматы тогда и только тогда, когда фрагменту $F$ не принадлежат особые цеепочки, а операция $\tau$ является правильной.

Доказательство. Сначала убедимся в необходимости требований условий леммы. Если $\tau$ не является правильной операцией, то легко заметить, что нетупиковый автомат $\tau(A, F)$ невозможно трансформировать в тупиковый преобразованиями из системы $T_{0}$.

Предположим, что $\tau$ - правильная операция, обозначим $\bar{F}_{2}$ образованный ее выполнением $s$-фрагмент, принадлежащий автомату $\tau(A, F)$, и пусть $B(A, F)$ - автомат, полученный из $\tau(A, F)$ выполнением $\varphi$-преобразования фрагмента $\bar{F}_{2}$. Если в автомате $F$ была особая цепочка, то в $B(A, F)$ будут эквивалентные состояния. Действительно, таковыми являются состояния $a$ и $b$, где $a$ - состояние, преемником которого является конец этой цепочки, а $b$ - копия этого конца, находящаяся во фрагменте $\varphi\left(\bar{F}_{2}\right)$. Легко видеть, что и в этом случае $\tau(A, F)$ нельзя трансформировать в тупиковый автомат преобразованиями из системы $T_{0}$.

Докажем достаточность условий леммы, сохранив только что введенные обозначения. Для этого установим, что автомат $B(A, F)$ тупиковый.

Состояния автомата $B(A, F)$ разобьем на множества $C_{0}, C_{1}, C_{2}, C_{3}$, полагая, что в $C_{0}$ входят $q$-состояния, принадлежащие нижней границе фрагмента $\varphi\left(\bar{F}_{2}\right)$, в $C_{1}-$ состояния из $F_{1}$, в $C_{2}-$ состояние из $F_{2}$, в $C_{3}$ - остальные состояния автомата $B(A, F)$.

Поскольку состояния из $C_{1} \cup C_{3}$ унаследованы из автомата $A$, а он тупиковый, все они попарно неэквивалентны. Состояния из $C_{2}$ также попарно неэквивалентны, ибо они были таковыми в $A$ и подверглись одинаковому воздействию выполненным $\varphi$-преобразованием фрагмента $\varphi\left(\bar{F}_{2}\right)$. Далее, в результате этого $\varphi$-преобразования стали попарно неэквивалентными состояния из $C_{1}$ и $C_{2}$, что обеспечено непринадлежностью фрагменту $F$ особых цепочек. Наконец, так как преемником состояний из $C_{0}$ являются состояния из $C_{3}$, а все они различны и попарно неэквивалентны, отсюда следует попарная неэквивалентность и состояний из $C_{0}$.

Итак, остается установить, что состояния из $C_{0}$ неэквивалентны состояниям из $C_{1}$, $C_{2}, C_{3}$, а состояния из $C_{2}$ неэквивалентны состояниям из $C_{3}$. Рассмотрим по отдельности все четыре пары множеств, действуя от противного. При этом предполагаемые эквивалентными состояния обозначим $a$ и $b$.

Случай 1. Пусть $a \in C_{0}, b \in C_{1}$. Если $b-$ это $q$-состояние, то состояния $a^{\prime}$ и $b^{\prime}$, являющиеся преемниками $a$ и $b$ соответственно, тоже эквивалентны. Состояние $a^{\prime}$ принадлежит $C_{3}$, а состояние $b^{\prime}-$ множеству $C_{1}$, ибо внутренними выходами фрагмента $F_{1}$ являются $p$-состояния. В силу попарной неэквивалентности состояний из $C_{1} \cup C_{3}$ эквивалентность $a^{\prime}$ и $b^{\prime}$ исключается.

Предположим, что $b$ - это $p$-состояние. Тогда в автомате $B(A, F)$ должен существовать фрагмент с нижней границей, содержащий состояние $b$, обозначим его $F^{\prime}$.

Убедимся в том, что $F^{\prime}$ является подфрагментом фрагмента $F_{1}$. Действительно, в ином случае граница фрагмента $F^{\prime}$ расположена за пределами внешних выходов фрагмента $F_{1}$ 
и $\varphi\left(\bar{F}_{2}\right)$, ибо они общие. Но тогда из эквивалентности состояний $a$ и $b$ вытекает, что должен существовать еще один фрагмент с нижней границей, для которого $F^{\prime}$ является строго вложенным в него подфрагментом. Продолжая эти рассуждения и дальше, мы придем к бесконечной череде фрагментов с нижней границей, что невозможно.

Итак, фрагмент $F^{\prime}$ - это подфрагмент фрагмента $F_{1}$, следовательно, и фрагмента $F$ в автомате $A$. Так как $A$ - перспективный автомат, фрагмент $F^{\prime}$ получен в нем успешным $\varphi$-преобразованием из некоторого $s$-фрагмента $F^{\prime \prime}$. Обратив это $\varphi$-преобразование $F^{\prime \prime}$ в $F^{\prime}$, мы получим автомат $\bar{A}$. Применяя доказательство, используемое в лемме 2 из [1], можно установить, что $\bar{A}-$ тупиковый автомат.

Автомату $\bar{A}$ принадлежат состояние $b$ и преемник состояния $a$, эквивалентность которых следует из предположения, что в $A$ были эквивалентны состояния $a$ и $b$. Поскольку $\bar{A}$ - тупиковый автомат, это заключение невозможно.

Случай 1 рассмотрен полностью.

Случай 2. Пусть $a \in C_{0}, b \in C_{2}$. Если $b-$ это $q$-состояние, то преемники $a$ и $b$ тоже эквивалентны; обозначим их $a^{\prime}$ и $b^{\prime}$ соответственно. Состояние $a^{\prime}$ принадлежит множеству $C_{3}$, а состояние $b^{\prime}-$ непременно множеству $C_{2}$ в силу того, что внутренними выходами фрагмента $F_{2}$ являются $p$-состояния. Этот случай подлежит рассмотрению в дальнейшем как случай 4.

Если $b$ - это $p$-состояние, то в автомате $B(A, F)$ должен существовать фрагмент с нижней границей, содержащий состояние $b$; обозначим его $F^{\prime}$. Ситуация, когда нижней границей фрагмента $F^{\prime}$ является нижняя граница фрагмента $\varphi\left(\bar{F}_{2}\right)$ невозможна, так как тогда во фрагменте $\bar{F}_{2}$ автомата $A$ должны быть эквивалентными состояние $b$ и преемник состояния $a$, являющийся внешним выходом фрагмента $\bar{F}_{2}$. Таким образом, нижняя граница фрагмента $F^{\prime}$ находится в $\bar{F}_{2}$, следовательно, если перейти к автомату $A$, то в $F$. Тогда полностью проходит рассуждение, изложенное в случае 1 и приводящее к желаемому противоречию.

Случай 2 исследован и требует анализа случая 4.

Случай 3. Пусть $a \in C_{0}, b \in C_{3}$. Если $b-$ это $q$-состояние, то опять-таки рассмотрим преемников $a$ и $b$, которых обозначим $a^{\prime}$ и $b^{\prime}$ соответственно. Они эквивалентны, при этом $a^{\prime}$ принадлежит множеству $C_{3}$, а $b^{\prime}-$ одному из множеств $C_{0}, C_{2}, C_{3}$; ситуация $b^{\prime} \in C_{1}$ исключается попарной неэквивалентностью состояний из $C_{1} \cup C_{3}$.

Ситуация $b^{\prime} \in C_{2}$ будет рассмотрена далее как случай 4 .

Если $b^{\prime} \in C_{3}$, то достаточно показать, что $a^{\prime} \neq b^{\prime}$, чтобы прийти к желаемому противоречию. Покажем это.

Равенство $a^{\prime}=b^{\prime}$ означает, что преемником состояния $b$ является внешний выход фрагмента $\varphi\left(\bar{F}_{2}\right)$. Но он является и внешним выходом фрагмента $\bar{F}_{2}$ в автомате $\tau(A, F)$; следовательно, при выполнении $\varphi$-преобразования состояние $b$ должно склеиваться с одним из состояний множества $C_{0}$, а это исключает принадлежность состояния $b$ множеству $C_{3}$.

Остается рассмотреть ситуацию, когда $b^{\prime} \in C_{0}$. Она означает, что в автомате $\tau(A, F)$ преемник состояния $b^{\prime}-$ это пассивный внешний выход фрагмента $\bar{F}_{2}$, и в результате $\varphi$-преобразования состояние $b^{\prime}$ попало в множество $C_{0}$. Прежде всего, отметим, что $b^{\prime} \neq a$. Действительно, при $b^{\prime}=a$ из эквивалентности $a$ и $b$ вытекает эквивалентность $b$ и $b^{\prime}$, чего не может быть, так как они оба являются $q$-состояниями.

Итак, $b^{\prime} \neq a$. Отыщем в автомате $\tau(A, F)$ прообраз состояния $b^{\prime}$, с одной стороны, и прообраз состояния $a^{\prime}$, с другой. Легко видеть, что они должны быть эквивалентными 
в $\tau(A, F)$, а последнее невозможно, так как оба принадлежат множеству $C_{3}$ и различны. Их различие следует из того, что преемником прообраза $b^{\prime}$ является пассивный внешний выход фрагмента $\bar{F}_{2}$, заведомо отличный от внешнего его выхода, каковым является прообраз состояния $a^{\prime}$; последнее обеспечивается неравенством $b^{\prime} \neq a$.

Обратимся теперь к ситуации, когда $b$ - это $p$-состояние. Тогда в автомате $B(A, F)$ должен существовать фрагмент с нижней границей, содержащий состояние $b$. Обозначим его $F^{\prime}$. Легко видеть, что состояния фрагмента $F^{\prime}$ и его внешние входы и выходы принадлежат множеству $C_{3}$. Так как $A-$ перспективный автомат, фрагмент $F^{\prime}$ получен успешным $\varphi$-преобразованием из $s$-фрагмента, состояния которого и внешние входы и выходы принадлежат множеству $C_{3}$; обозначим этот фрагмент $F^{\prime \prime}$. Обратив в автомате $A$ $\varphi$-преобразование $F^{\prime \prime}$ в $F^{\prime}$, мы получим автомат $\bar{A}$, тупиковость которого обеспечивается доказательствами, проведенными в лемме 2 из [1]. В автомате $\bar{A}$ состояние $b$ должно быть эквивалентным преемнику состояния $a$, что противоречит его тупиковости, так как эти состояния различны.

Итак, случай 3 исследован и требует рассмотрения случая 4.

Случай 4. Пусть $a \in C_{2}, b \in C_{3}$. Пусть $b-$ это $q$-состояние. Обозначим $b^{\prime}$ его преемника. Из эквивалентности $a$ и $b$ следует, что в автомате $\tau(A, F)$ состояния $b^{\prime}$ и $a$ должны быть эквивалентны.

Состояние $b^{\prime}$ в автомате $\tau(A, F)$ принадлежит одному из множеств $C_{2}$ и $C_{3}$, ибо ситуация $b^{\prime} \in C_{3}$ исключается правильностью операции $\tau$.

Если $b^{\prime} \in C_{2}$, то $b$ находится в верхней границе фрагмента $\bar{F}_{2}$, и эквивалентность состояний $b^{\prime}$ и $a$ исключена.

Если же $b^{\prime} \in C_{3}$, то рассмотрим в $C_{1}$ состояние $\bar{a}$, эквивалентное состоянию $a$. Состояния $\bar{a}$ и $b^{\prime}$ должны быть эквивалентны в $\tau(A, F)$, но их принадлежность множествам $C_{1}$ и $C_{3}$ исключает это.

Предположим теперь, что $b-$ это $p$-состояние.

Если $a$ - это $q$-состояние, то в автомате $B(A, F)$ должен существовать фрагмент с нижней границей, содержащий состояние $b$ и такой, что его состояния и внешние входы и выходы принадлежат $C_{3}$. Обозначим его $F^{\prime}$. Подобно тому, как это делалось в случае 3 , обозначим $F^{\prime \prime}$ тот $s$-фрагмент, из которого успешным $\varphi$-преобразованием получен фрагмент $F^{\prime}$, и обратим это $\varphi$-преобразование, обозначив $\bar{A}$ полученный таким образом автомат; он, как уже отмечалось, является тупиковым. Из предполагаемой эквивалентности состояний $a$ и $b$ в автомате $B(A, F)$ следует их эквивалентность в автомате $\bar{A}$, а это противоречит его тупиковости.

Рассмотрим, наконец, ситуацию, когда $a$ и $b$ - это $p$-состояния. Возьмем кратчайшие пути из $a$ и $b$ в финальное состояние, которые несут эквивалентные цепочки (последнее понимается аналогично тому, как оценивалась эквивалентность путей через автоматы); обозначим эти пути $w_{a}$ и $w_{b}$ соответственно. Установим, что в них существует пара равноудаленных от их начала позиций, одна из которых занята $p$-состоянием, а другая $q$-состоянием. Действительно, в противном случае, просматривая пути $w_{a}$ и $w_{b}$ от финального состояния к их началу, легко установить, что они совпадают, пока проходят по состояниям из $C_{3}$. Но, достигнув состояния, принадлежащего множеству $C_{0}$, путь $w_{a}$ обязан совпадать и в этом состоянии с путем $w_{b}$, так как в $B(A, F)$ нет различных $q$-состояний, имеющих общего преемника. А такое совпадение невозможно.

Проведенное исследование доказывает, что в путях $w_{a}, w_{b}$ имеются разнотипные и эквивалентные состояния, первое из которых принадлежит либо множеству $C_{2}$, либо множеству $C_{0}$, а второе - множеству $C_{3}$. Эти ситуации уже разобраны, и установлено, 
что они приводят к противоречию.

Случай 4 рассмотрен полностью.

В итоге нами показано, что при выполнении условий леммы 2 автомат $B(A, F)$ является тупиковым, то есть утверждение леммы справедливо. Лемма 2 доказана.

\section{5. Процедура отыскания всех минимальных автоматов в классе эквивалентности множества $\bar{M}$}

Предлагаемая процедура основана на ряде предварительно устанавливаемых фактов. Изложим их, полагая, что рассматривается класс $K$ эквивалентности из $\bar{M}$.

Пусть $F$ - фрагмент перспективного автомата $A$. Назовем $F$ корректным, если он допускает правильную операцию $\tau$ и не содержит особых цепочек. В случае, когда $F-$ корректный фрагмент, срез $\tau(A, F)$ называем допустимым.

Лемма 3. Для любого допустимого среза класса эквивалентности $K$ сущеествует проиедура построения минимального в срезе автомата.

Доказательство. Пусть $\tau(A, F)$ - допустимый срез. В тупиковом автомате $B(A, F)$ все принадлежащие ему $s$-фрагменты классифицируются по признаку, унаследован ли этот фрагмент из автомата $A$ или он возник в результате операции $\tau$.

Очевидно, что $s$-фрагменты первого типа не допускают успешных $\varphi$-преобразований. Если не существуют фрагменты второго типа, то автомат $B(A, F)$ является минимальным в срезе $\tau(A, F)$.

В противном случае рассмотрим фрагменты второго типа. Легко видеть, что, какими бы ни были два из них, один вложен в другой, и в каждый из них вложен фрагмент $\varphi\left(\bar{F}_{2}\right)$. Применение $\varphi$-преобразования к любому из этих фрагментов транслирует тупиковый автомат в тупиковый. Доказательство этого утверждения представляет собой повторение доказательства леммы 2 , если в нем множество $C_{0}$ полагать состоящим не только из нижней границы фрагмента $\varphi\left(\bar{F}_{2}\right)$, но и из нижней границы фрагмента, полученного примененным $\varphi$-преобразованием. Более того, тупиковость сохраняется и в случае нескольких $\varphi$-преобразований, выполненных для $s$-фрагментов второго типа.

Если среди $s$-фрагментов второго типа отсутствуют обеспечивающие успешное $\varphi$-преобразование, то по-прежнему автомат $B(A, F)$ будет минимальным в срезе $\tau(A, F)$. Иначе для получения минимального автомата необходимо выполнить все успешные $\varphi$-преобразования фрагментов второго типа. Нетрудно заметить, что порядок их выполнения безразличен, то есть не влияет на окончательный результат. Последний и будет минимальным автоматом в срезе $\tau(A, F)$.

Лемма 3 доказана.

Автомат, полученный описанной в лемме 3 процедурой, назовем стержневым в срезе $\tau(A, F)$ и будем обозначать $D(A, F)$. Очевидно, что $D(A, F)$ - перспективный автомат.

Следствие 1. Все минимальные автоматы в допустимом срезе $\tau(A, F)$, отличные от $D(A, F)$, получаются из него устойчивыми ф-преобразованиями, если таковые имеются.

Лемма 4. Свойство корректности фрагмента распознаваемо.

Доказательство. Пусть $F-$ фрагмент перспективного автомата. Полагаем, что все внутренние выходы $F$ - это $p$-состояния, и $F$ не содержит особых цепочек. Эти требования проверяемы. Таким образом, нужно установить, каковы требования, предъявляемые к 
внутренним входам фрагмента $F$, обеспечивающие, во-первых, возможность его копирования (в копии каждое состояние должно лежать на пути из входа в выход) и, во-вторых, максимальность $s$-фрагмента, индуцируемого операцией $\tau$.

Легко убедиться в том, что по предъявляемым требованиям внутренние входы $F$ разбиваются на три типа:

тип 1: вход является преемником нескольких состояний, одно из которых непременно $q$-состояние;

тип 2: вход является преемником только $q$-состояния и достижим в $F$ из некоторого входа типа 1;

тип 3: вход - это $q$-состояние, и он достижим в $F$ из некоторого входа типа 1.

Очевидно, что эти требования проверяются эффективно. Лемма 4 доказана.

Пусть $\tau(A, F)$ - допустимый срез класса $K$. Последовательность преобразований, посредством которых из перспективного автомата $A$ получен стержневой автомат $D(A, F)$, будем именовать операцией $\rho$ над $F$ в $A$.

Возьмем корректный фрагмент $F^{\prime}$ автомата $A$, отличный от корректного фрагмента $F$. Говорим, что по выполнении операции $\rho$ над $F$ в $A$ образом фрагмента $F^{\prime}$ в автомате $D(A, F)$ является наименьшего размера его фрагмент, который содержит все состояния, принадлежащие ранее фрагменту $F^{\prime}$.

Предложение 2. По выполнении операции $\rho$ над $F$ в $A$ автомат $D(A, F)$ содержит образы всех корректных фрагментов автомата $A$, и этим образам принадлежат все корректные фрагменты автомата $D(A, F)$.

Справедливость предложения 2 очевидна.

По определению, операция $\rho$ над $F$ в $A$ разрушает корректный фрагмент $F^{\prime}$ автомата $A$, если его образ в $D(A, F)$ не является корректным фрагментом.

Предложение 3. Для любых двух корректных фрагментов $F$, $F^{\prime}$ перспективного автомата распознаваемо, разрушает ли операция $\rho$ над $F$ в А фрагмент $F^{\prime}$.

Действительно, по выполнении операции $\rho$ над $F$ в $A$ однозначно определяется образ фрагмента $F^{\prime}$ в $D(A, F)$. Всегда легко установить, удовлетворяет ли он требованиям, изложенным в лемме 4.

Приведем пример того, что разрушения корректных фрагментов возможны. Предположим, что внутренние входы фрагментов $F$ и $F^{\prime}$ пересекаются. Тогда корректность образа фрагмента $F^{\prime}$ имеет место только в случае выполнимости следующих требований: в фрагменте $F^{\prime}$ должен быть вход типа 1 , не принадлежащий фрагменту $F$; все внутренние входы, являющиеся общими для $F$ и $F^{\prime}$, должны быть достижимы из собственных внутренних входов фрагмента $F^{\prime}$, и если общий вход имеет тип 1 , то он обязан быть $q$-состоянием. При нарушении одного из этих требований будет иметь место факт разрушения фрагмента $F^{\prime}$ операцией $\rho$ над $F$ в $A$.

Пусть $F_{1}$ и $F_{2}$ - корректные фрагменты перспективного автомата $A$.

Легко доказывается следующее утверждение.

Предложение 4. Если для $i=1,2$ операчия $\rho$ над $F_{i}$ в $A$ не разрушает фрагмент $F_{3-i}$, то автомат, полученный операчией $\rho$ над образом фрагмента $F_{2}$ в автомате $D\left(A, F_{1}\right)$, изоморфен автомату, полученному операцией $\rho$ над образом $F_{1}$ в $D\left(A, F_{2}\right)$. 
Это предложение, в сущности, говорит о перестановочности операции $\rho$ над фрагментами $F_{1}, F_{2}$.

Покрытием перспективного автомата $A$ назовем множество его корректных фрагментов, в котором ни один из них не разрушает другой при выполнении над ним операции $\rho$ в $A$. Покрытие называется полным, если добавление к нему любого корректного фрагмента автомата $A$ нарушает его статус покрытия.

Предложение 5. Каким бы ни был фрагмент $F$ из покрытия перспективного автомата A, образы всех остальных фрагментов из этого покрытия, принадлежашие автомату $D(A, F)$, составляют покрытие последнего; при полноте покрытия автомата $A$ полным является и покрытие автомата $D(A, F)$.

Справедливость предложения 5 очевидна.

Наконец, докажем теорему 1.

Теорема 1. Каким бы ни был класс эквивалентности $K$ из множества $\bar{M}$, существует процедура построения всех минимальных автоматов в $\mathrm{K}$.

Доказательство. Нами предлагается процедура, предписывающая два этапа действий. На первом из них определяются минимальные в $K$ автоматы, являющиеся стержневыми в своих срезах класса $K$. Пусть они составляют множество $\mathscr{L}$. Второй этап состоит в том, что для каждого автомата из $\mathscr{L}$ применением к нему устойчивых $\varphi$-преобразований конструируются все остальные минимальные автоматы в срезе, которому принадлежит выбранный стержневой автомат.

Таким образом, предстоит описать лишь первый этап процедуры и убедиться в его корректности.

Текущим в процедуре объявляется список $S$, состоящий из автоматов, которые на данный момент выполнения процедуры претендуют на минимальность в $K$. Условимся обозначать $l$ размер автоматов из $S$ (они равновелики). По числу $l$ определяется размер корректных фрагментов, подлежащих далее рассмотрению.

В начальный момент $S$ состоит из одного элемента - основного автомата $A_{0}$, то есть стержневого автомата главного среза класса $K$, а $l$ определяется его размером. Для этого процедурой используется описанное в [1] построение основного автомата.

Далее для автомата $A_{0}$ строятся все полные его покрытия корректными фрагментами допустимого размера, устанавливаемого числом $l$. Полные покрытия автомата $A_{0}$ составляют конечное множество $R$. Эти покрытия рассматриваются друг за другом, причем порядок их рассмотрения безразличен.

Пусть исследуемое покрытие $Q$ из $R$, список $S$ и число $l$ определены. Это исследование распадается на отдельные шаги, на каждом из которых выполняется операция $\rho$ над корректным фрагментом перспективного автомата, рассматриваемого на данном шаге.

Описание отдельного шага сделаем на примере первого, поскольку все они идентичны.

Итак, полагаем множество $Q$ упорядоченным, и пусть $F-$ первый принадлежащий $Q$ фрагмент автомата $A_{0}$. Выполняемый шаг начинается применением операции $\rho$ к $F$, после чего размер автомата $D\left(A_{0}, F\right)$ (обозначим его $\left.l^{\prime}\right)$ сравнивается с размером автоматов в списке $S$, то есть с числом $l$.

Если $l^{\prime}>l$, то на следующем шаге будет рассматриваться автомат $A_{0}$; список $S$ и число $l$ не изменяются.

Если $l^{\prime}=l$, то на следующем шаге будет рассматриваться автомат $D\left(A_{0}, F\right)$; он вносится в список $S$, число $l$ сохраняется.

Если $l^{\prime}<l$, то на следующем шаге рассмотрению подлежит автомат $D\left(A_{0}, F\right)$; список $S$ очищается, в него вносится автомат $D\left(A_{0}, F\right)$; число $l$ полагается равным $l^{\prime}$. 
Во всех трех случаях, если множество $Q$ не исчерпано, то следующий шаг состоит в исследовании очередного фрагмента из $Q$; он остается неизменным при $l^{\prime}>l$ и заменяется его образом в автомате $D\left(A_{0}, F\right)$ при $l^{\prime}=l$ и при $l^{\prime}<l$; учитывается здесь и новая планка $l$.

По исчерпании покрытия $Q$ процедурой обрабатывается другое из $R$, и так до тех пор, пока не будут рассмотрены все покрытия. Тогда процедура считает завершенным первый этап своей работы.

Корректность описанного этапа основана на том, что по выполнении любого шага на следующем рассматривается непременно перспективный автомат, для которого справедливы леммы 2 и 3. Предложения 2-5 обосновывают правильность предложенного разбиения этапа на отдельные шаги. Таким образом, в список $S$ действительно попадают все наименьшего размера стержневые автоматы, принадлежащие срезам класса $K$.

Теорема 1 доказана.

Следствие 2. В множестве М решена проблема минимизаџии.

Действительно в $\bar{M}$ она решена, а теоремой 2, доказанной в [1], проблема минимизации в $M$ сводится к проблеме минимизации в $\bar{M}$.

\section{Список литературы}

1. Подловченко Р. И., Хачатрян В. Е., Минимальность и тупиковость многоленточных автоматов. Дискретная математика (2008) 20, №2, 1-25.

2. Подловченко Р. И., К вопросу об эквивалентных преобразованиях алгоритмов и программ. $M a-$ тематические вопросы кибернетики (2000) 9, 25-36.

3. Хачатрян В. Е., Решение обобщенной проблемы минимизации для двухленточных автоматов с одной фиксированной лентой. Доклады РАН (2006) 411, №3, 314-318.

Статья поступила 2.03.2010. 